\title{
On the Fermionic $p$-adic Integral Representation of Bernstein Polynomials Associated with Euler Numbers and Polynomials
}

\author{
T. $\operatorname{Kim}^{1}{ }^{1}$ J. Choi, ${ }^{1}$ Y. H. $\operatorname{Kim}^{1}{ }^{1}$ and C. S. Ryoo $^{2}$ \\ ${ }^{1}$ Division of General Education-Mathematics, Kwangwoon University, Seoul 139-701, Republic of Korea \\ 2 Department of Mathematics, Hannam University, Daejeon 306-791, Republic of Korea
}

Correspondence should be addressed to T. Kim, tkkim@kw.ac.kr

Received 30 August 2010; Accepted 3 December 2010

Academic Editor: Paolo E. Ricci

Copyright (C) 2010 T. Kim et al. This is an open access article distributed under the Creative Commons Attribution License, which permits unrestricted use, distribution, and reproduction in any medium, provided the original work is properly cited.

The purpose of this paper is to give some properties of several Bernstein type polynomials to represent the fermionic $p$-adic integral on $\mathbb{Z}_{p}$. From these properties, we derive some interesting identities on the Euler numbers and polynomials.

\section{Introduction}

Throughout this paper, let $p$ be an odd prime number. The symbol, $\mathbb{Z}_{p}, \mathbb{Q}_{p}$, and $\mathbb{C}_{p}$ denote the ring of $p$-adic integers, the field of $p$-adic rational numbers, the complex number field and the completion of algebraic closure of $\mathbb{Q}_{p}$, respectively.

Let $\mathbb{N}$ be the set of natural numbers and $\mathbb{Z}_{+}=\mathbb{N} \cup\{0\}$. Let $v_{p}$ be the normalized exponential valuation of $\mathbb{C}_{p}$ with $|p|_{p}=p^{-v_{p}(p)}=1 / p$. Note that $\mathbb{Z}_{p}=\left\{\left.x|| x\right|_{p} \leq 1\right\}=$ $\lim _{\overleftarrow{N}} \mathbb{Z} / p^{N} \mathbb{Z}_{p}$.

When one talks of $q$-extension, $q$ is variously considered as an indeterminate, a complex number $q \in \mathbb{C}$, or $p$-adic number $q \in \mathbb{C}_{p}$. If $q \in \mathbb{C}$, we normally assume $|q|<1$, and if $q \in \mathbb{C}_{p}$, we always assume $|1-q|_{p}<1$.

We say that $f$ is uniformly differentiable function at a point $a \in \mathbb{Z}_{p}$ and write $f \in U D\left(\mathbb{Z}_{p}\right)$, if the difference quotient $F_{f}(x, y)=(f(x)-f(y)) /(x-y)$ has a limit $f^{\prime}(a)$ as $(x, y) \rightarrow(a, a)$. For $f \in U D\left(\mathbb{Z}_{p}\right)$, the fermionic $p$-adic $q$-integral on $\mathbb{Z}_{p}$ is defined as

$$
I_{-q}(f)=\int_{\mathbb{Z}_{p}} f(x) d \mu_{-q}(x)=\lim _{N \rightarrow \infty} \frac{1+q}{1+q^{p^{N}}} \sum_{x=0}^{p^{N}-1} f(x)(-q)^{x},
$$


(see [1]). In the special case $q=1$ in (1.1), the integral

$$
I_{-1}(f)=\int_{\mathbb{Z}_{p}} f(x) d \mu_{-1}(x)
$$

is called the fermionic $p$-adic invariant integral on $\mathbb{Z}_{p}$ (see [2]). From (1.2), we note

$$
I_{-1}\left(f_{1}\right)=-I_{-1}(f)+2 f(0),
$$

where $f_{1}(x)=f(x+1)$.

Moreover, for $n \in \mathbb{N}$, let $f_{n}(x)=f(x+n)$. Then we note that

$$
I_{-1}\left(f_{n}\right)=(-1)^{n} I_{-1}(f)+2 \sum_{l=0}^{n-1}(-1)^{n-1-l} f(l)
$$

It is well known that the Euler polynomials are defined by

$$
\frac{2}{e^{t}+1} e^{x t}=\sum_{n=0}^{\infty} E_{n}(x) \frac{t^{n}}{n !}
$$

(see [1-15]). In the special case, $x=0$, and $E_{n}(0)=E_{n}$ are called the $n$th Euler numbers.

Let $f(x)=e^{t x}$. Then, by (1.3), (1.4), and (1.5), we see that

$$
\int_{\mathbb{Z}_{p}} e^{(x+y) t} d \mu_{-1}(y)=\frac{2}{e^{t}+1} e^{x t}=\sum_{n=0}^{\infty} E_{n}(x) \frac{t^{n}}{n !} .
$$

Let $C[0,1]$ denote the set of continuous functions on $[0,1]$. For $f \in C[0,1]$, Bernstein introduced the following well-known linear positive operator in the field of real numbers $\mathbb{R}$ :

$$
\mathbb{B}_{n}(f: x)=\sum_{k=0}^{n} f\left(\frac{k}{n}\right)\left(\begin{array}{l}
n \\
k
\end{array}\right) x^{k}(1-x)^{n-k}=\sum_{k=0}^{n} f\left(\frac{k}{n}\right) B_{k, n}(x),
$$

where $\left(\begin{array}{c}n \\ k\end{array}\right)=(n(n-1) \cdots(n-k+1)) / k !=n ! / k !(n-k) !($ see $[3,4,7,10,11,14])$. Here, $\mathbb{B}_{n}(f: x)$ is called the Bernstein operator of order $n$ for $f$.

For $k, n \in \mathbb{Z}_{+}$, the Bernstein polynomial of degree $n$ is defined by

$$
B_{k, n}(x)=\left(\begin{array}{l}
n \\
k
\end{array}\right) x^{k}(1-x)^{n-k}, \quad \text { for } x \in[0,1] \text {. }
$$

For example, $B_{0,1}(x)=1-x, B_{1,1}(x)=x, B_{0,2}(x)=(1-x)^{2}, B_{1,2}(x)=2 x-2 x^{2}, B_{2,2}(x)=x^{2}, \ldots$, and $B_{k, n}(x)=0$ for $n<k, B_{\mathrm{k}, n}(x)=B_{n-k, n}(1-x)$.

In this paper, we study the properties of Bernstein polynomials in the $p$-adic number field. For $f \in U D\left(\mathbb{Z}_{p}\right)$, we give some properties of several type Bernstein polynomials 
to represent the fermionic $p$-adic invariant integral on $\mathbb{Z}_{p}$. From those properties, we derive some interesting identities on the Euler polynomials.

\section{Fermionic $p$-adic Integral Representation of Bernstein Polynomials}

By (1.5) and (1.6), we see that

$$
\frac{2}{e^{t}+1} e^{(1-x) t}=\sum_{n=0}^{\infty} E_{n}(1-x) \frac{t^{n}}{n !}
$$

We also have that

$$
\frac{2}{e^{t}+1} e^{(1-x) t}=\frac{2}{1+e^{-t}} e^{-x t}=\sum_{n=0}^{\infty} E_{n}(x) \frac{(-1)^{n}}{n !} t^{n}
$$

From (2.1) and (2.2), we note that $E_{n}(1-x)=(-1)^{n} E_{n}(x)$. It is easy to show that

$$
E_{n}(2)=2-\sum_{l=0}^{n}\left(\begin{array}{l}
n \\
l
\end{array}\right) E_{l}=2+E_{n}, \quad \text { for } n>0 .
$$

By (1.5), (1.6), (2.1), (2.2), and (2.3), we see that for $n>0$,

$$
\begin{aligned}
\int_{\mathbb{Z}_{p}}(1-x)^{n} d \mu_{-1}(x) & =(-1)^{n} \int_{\mathbb{Z}_{p}}(x-1)^{n} d \mu_{-1}(x)=\int_{\mathbb{Z}_{p}}(x+2)^{n} d \mu_{-1}(x) \\
& =2+\int_{\mathbb{Z}_{p}} x^{n} d \mu_{-1}(x) .
\end{aligned}
$$

Therefore, we obtain the following theorem.

Theorem 2.1. For $n \in \mathbb{N}$, one has

$$
\int_{\mathbb{Z}_{p}}(1-x)^{n} d \mu_{-1}(x)=2+\int_{\mathbb{Z}_{p}} x^{n} d \mu_{-1}(x)
$$

Theorem 2.1 is important to derive our main result in this paper. 
Taking the fermionic $p$-adic integral on $\mathbb{Z}_{p}$ for one Bernstein polynomial in (1.8), we get

$$
\begin{aligned}
\int_{\mathbb{Z}_{p}} B_{k, n}(x) d \mu_{-1}(x) & =\int_{\mathbb{Z}_{p}}\left(\begin{array}{l}
n \\
k
\end{array}\right) x^{k}(1-x)^{n-k} d \mu_{-1}(x) \\
& =\left(\begin{array}{l}
n \\
k
\end{array}\right) \sum_{j=0}^{n-k}\left(\begin{array}{c}
n-k \\
j
\end{array}\right)(-1)^{n-k-j} \int_{\mathbb{Z}_{p}} x^{n-j} d \mu_{-1}(x) \\
& =\left(\begin{array}{l}
n \\
k
\end{array}\right) \sum_{j=0}^{n-k}\left(\begin{array}{c}
n-k \\
j
\end{array}\right)(-1)^{n-k-j} E_{n-j} \\
& =\left(\begin{array}{l}
n \\
k
\end{array}\right) \sum_{j=0}^{n-k}\left(\begin{array}{c}
n-k \\
j
\end{array}\right)(-1)^{j} E_{k+j} .
\end{aligned}
$$

Therefore, we obtain the following proposition.

Proposition 2.2. For $k, n \in \mathbb{Z}_{+}$one is

$$
\int_{\mathbb{Z}_{p}} B_{k, n}(x) d \mu_{-1}(x)=\left(\begin{array}{l}
n \\
k
\end{array}\right) \sum_{j=0}^{n-k}\left(\begin{array}{c}
n-k \\
j
\end{array}\right)(-1)^{j} E_{k+j} .
$$

It is known that $B_{k, n}(x)=B_{n-k, n}(1-x)$. Thus, one has

$$
\begin{aligned}
\int_{\mathbb{Z}_{p}} B_{k, n}(x) d \mu_{-1}(x) & =\int_{\mathbb{Z}_{p}} B_{n-k, n}(1-x) d \mu_{-1}(x) \\
& =\left(\begin{array}{c}
n \\
n-k
\end{array}\right) \sum_{j=0}^{k}\left(\begin{array}{l}
k \\
j
\end{array}\right)(-1)^{k-j} \int_{\mathbb{Z}_{p}}(1-x)^{n-j} d \mu_{-1}(x) .
\end{aligned}
$$

By (2.8) and Theorem 2.1, we see that for $n>k$,

$$
\begin{aligned}
\int_{\mathbb{Z}_{p}} B_{k, n}(x) d \mu_{-1}(x) & =\left(\begin{array}{l}
n \\
k
\end{array}\right) \sum_{j=0}^{k}\left(\begin{array}{l}
k \\
j
\end{array}\right)(-1)^{k-j}\left(2+\int_{\mathbb{Z}_{p}} x^{n-j} d \mu_{-1}(x)\right) \\
& =\left(\begin{array}{l}
n \\
k
\end{array}\right) \sum_{j=0}^{\mathrm{k}}\left(\begin{array}{l}
k \\
j
\end{array}\right)(-1)^{k-j}\left(2+E_{n-j}\right) \\
& = \begin{cases}2+E_{n} & \text { if } k=0, \\
\left(\begin{array}{l}
n \\
k
\end{array}\right) \sum_{j=0}^{k}\left(\begin{array}{l}
k \\
j
\end{array}\right)(-1)^{k-j} E_{n-j} & \text { if } k>0 .\end{cases}
\end{aligned}
$$

From (2.9), we obtain the following theorem. 
Theorem 2.3. For $n, k \in \mathbb{Z}_{+}$with $n>k$, we have

$$
\int_{\mathbb{Z}_{p}} B_{k, n}(x) d \mu_{-1}(x)= \begin{cases}2+E_{n} & \text { if } k=0, \\
\left(\begin{array}{l}
n \\
k
\end{array}\right) \sum_{j=0}^{k}\left(\begin{array}{l}
k \\
j
\end{array}\right)(-1)^{k-j} E_{n-j} & \text { if } k>0 .\end{cases}
$$

By Proposition 2.2 and Theorem 2.3, we obtain the following corollary.

Corollary 2.4. For $n, k \in \mathbb{Z}_{+}$with $n>k$, we have

$$
\sum_{j=0}^{n-k}\left(\begin{array}{c}
n-k \\
j
\end{array}\right)(-1)^{j} E_{k+j}= \begin{cases}2+E_{n} & \text { if } k=0 \\
\sum_{j=0}^{k}\left(\begin{array}{l}
k \\
j
\end{array}\right)(-1)^{k-j} E_{n-j} & \text { if } k>0 .\end{cases}
$$

For $m, n, k \in \mathbb{Z}_{+}$with $m+n>2 k$, fermionic $p$-adic invariant integral for multiplication of two Bernstein polynomials on $\mathbb{Z}_{p}$ can be given by the following relation:

$$
\begin{aligned}
\int_{\mathbb{Z}_{p}} B_{k, n}(x) B_{k, m}(x) d \mu_{-1}(x) & =\int_{\mathbb{Z}_{p}}\left(\begin{array}{l}
n \\
k
\end{array}\right) x^{k}(1-x)^{n-k}\left(\begin{array}{l}
m \\
k
\end{array}\right) x^{k}(1-x)^{m-k} d \mu_{-1}(x) \\
& =\left(\begin{array}{l}
n \\
k
\end{array}\right)\left(\begin{array}{l}
m \\
k
\end{array}\right) \int_{\mathbb{Z}_{p}} x^{2 k}(1-x)^{n+m-2 k} d \mu_{-1}(x) \\
& =\left(\begin{array}{l}
n \\
k
\end{array}\right)\left(\begin{array}{l}
m \\
k
\end{array}\right) \sum_{j=0}^{2 k}\left(\begin{array}{c}
2 k \\
j
\end{array}\right)(-1)^{j+2 k} \int_{\mathbb{Z}_{p}}(1-x)^{n+m-j} d \mu_{-1}(x) \\
& =\left(\begin{array}{l}
n \\
k
\end{array}\right)\left(\begin{array}{l}
m \\
k
\end{array}\right) \sum_{j=0}^{2 k}\left(\begin{array}{c}
2 k \\
j
\end{array}\right)(-1)^{j+2 k}\left(2+\int_{\mathbb{Z}_{p}} x^{n+m-j} d \mu_{-1}(x)\right) \\
& =\left\{\begin{array}{c}
2+E_{n+m} k=0, \\
\left(\begin{array}{l}
n \\
k
\end{array}\right)\left(\begin{array}{l}
m \\
k
\end{array}\right) \sum_{j=0}^{2 k}\left(\begin{array}{c}
2 k \\
j
\end{array}\right)^{(-1)^{j+2 k} E_{n+m-j} \quad \text { if } k>0 .}
\end{array}\right.
\end{aligned}
$$


Therefore, we obtain the following theorem.

Theorem 2.5. For $m, n, k \in \mathbb{Z}_{+}$with $m+n>2 k$, one has

$$
\int_{\mathbb{Z}_{p}} B_{k, n}(x) B_{k, m}(x) d \mu_{-1}(x)= \begin{cases}2+E_{n+m} & \text { if } k=0, \\
\left(\begin{array}{l}
n \\
k
\end{array}\right)\left(\begin{array}{l}
m \\
k
\end{array}\right) \sum_{j=0}^{2 k}\left(\begin{array}{c}
2 k \\
j
\end{array}\right)(-1)^{j+2 k} E_{n+m-j} & \text { if } k>0 .\end{cases}
$$

For $m, n, k \in \mathbb{Z}_{+}$, one has

$$
\begin{aligned}
\int_{\mathbb{Z}_{p}} B_{k, n}(x) B_{k, m}(x) d \mu_{-1}(x) & =\left(\begin{array}{l}
n \\
k
\end{array}\right)\left(\begin{array}{l}
m \\
k
\end{array}\right) \int_{\mathbb{Z}_{p}} x^{2 k}(1-x)^{n+m-2 k} d \mu_{-1}(x) \\
& =\left(\begin{array}{l}
n \\
k
\end{array}\right)\left(\begin{array}{l}
m \\
k
\end{array}\right) \sum_{j=0}^{n+m-2 k}\left(\begin{array}{c}
n+m-2 k \\
j
\end{array}\right)(-1)^{j} \int_{\mathbb{Z}_{p}} x^{j+2 k} d \mu_{-1}(x) \\
& =\left(\begin{array}{l}
n \\
k
\end{array}\right)\left(\begin{array}{l}
m \\
k
\end{array}\right) \sum_{j=0}^{n+m-2 k}\left(\begin{array}{c}
n+m-2 k \\
j
\end{array}\right)(-1)^{j} E_{j+2 k} .
\end{aligned}
$$

Thus, we obtain the following proposition.

Proposition 2.6. For $m, n, k \in \mathbb{Z}_{+}$one has

$$
\int_{\mathbb{Z}_{p}} B_{k, n}(x) B_{k, m}(x) d \mu_{-1}(x)=\left(\begin{array}{l}
n \\
k
\end{array}\right)\left(\begin{array}{l}
m \\
k
\end{array}\right) \sum_{j=0}^{n+m-2 k}\left(\begin{array}{c}
n+m-2 k \\
j
\end{array}\right)(-1)^{j} E_{j+2 k} .
$$

By Theorem 2.5 and Proposition 2.6, we obtain the following corollary.

Corollary 2.7. For $m, n, k \in \mathbb{Z}_{+}$with $m+n>2 k$, one has

$$
\sum_{j=0}^{n+m-2 k}\left(\begin{array}{cl}
n+m-2 k \\
j
\end{array}\right)(-1)^{j} E_{j+2 k}= \begin{cases}2+E_{n+m} & \text { if } k=0 \\
\sum_{j=0}^{2 k}\left(\begin{array}{c}
2 k \\
j
\end{array}\right)(-1)^{j+2 k} E_{n+m-j} & \text { if } k>0 .\end{cases}
$$


Journal of Inequalities and Applications

In the same manner, multiplication of three Bernstein polynomials can be given by the following relation:

$$
\begin{aligned}
\int_{\mathbb{Z}_{p}} B_{k, n}(x) B_{k, m}(x) B_{k, s}(x) d \mu_{-1}(x) \\
=\left(\begin{array}{l}
n \\
k
\end{array}\right)\left(\begin{array}{l}
m \\
k
\end{array}\right)\left(\begin{array}{l}
s \\
k
\end{array}\right) \sum_{j=0}^{n+m+s-3 k}\left(\begin{array}{c}
n+m+s-3 k \\
j
\end{array}\right)(-1)^{j} \int_{\mathbb{Z}_{p}} x^{j+3 k} d \mu_{-1}(x) \\
=\left(\begin{array}{l}
n \\
k
\end{array}\right)\left(\begin{array}{l}
m \\
k
\end{array}\right)\left(\begin{array}{l}
s \\
k
\end{array}\right) \sum_{j=0}^{n+m+s-3 k}\left(\begin{array}{c}
n+m+s-3 k \\
j
\end{array}\right)(-1)^{j} E_{j+3 k},
\end{aligned}
$$

where $m, n, s, k \in \mathbb{Z}_{+}$with $m+n+s>3 k$. see that

For $m, n, s, k \in \mathbb{Z}_{+}$with $m+n+s>3 k$, by the symmetry of Bernstein polynomals, we

$$
\begin{aligned}
& \int_{\mathbb{Z}_{p}} B_{k, n}(x) B_{k, m}(x) B_{k, s}(x) d \mu_{-1}(x) \\
& \quad=\left(\begin{array}{l}
n \\
k
\end{array}\right)\left(\begin{array}{l}
m \\
k
\end{array}\right)\left(\begin{array}{l}
s \\
k
\end{array}\right) \sum_{j=0}^{3 k}\left(\begin{array}{c}
3 k \\
j
\end{array}\right)(-1)^{3 k-j} \int_{\mathbb{Z}_{p}}(1-x)^{n+m+s-j} d \mu_{-1}(x) \\
& \quad=\left(\begin{array}{l}
n \\
k
\end{array}\right)\left(\begin{array}{l}
m \\
k
\end{array}\right)\left(\begin{array}{l}
s \\
k
\end{array}\right) \sum_{j=0}^{3 k}\left(\begin{array}{c}
3 k \\
j
\end{array}\right)(-1)^{3 k-j}\left(2+\int_{\mathbb{Z}_{p}} x^{n+m+s-j} \mu_{-1}(x)\right) \\
& \quad=\left\{\begin{array}{l}
2+E_{n+m+s} \\
\left(\begin{array}{l}
n \\
k
\end{array}\right)\left(\begin{array}{l}
m \\
k
\end{array}\right)\left(\begin{array}{l}
s \\
k
\end{array}\right) \sum_{j=0}^{3 k}\left(\begin{array}{c}
3 k \\
j
\end{array}\right)(-1)^{3 k-j} E_{n+m+s-j} \text { if } k>0 .
\end{array}\right.
\end{aligned}
$$

Therefore, we obtain the following theorem.

Theorem 2.8. For $m, n, s, k \in \mathbb{Z}_{+}$with $m+n+s>3 k$, one has

$$
\begin{aligned}
& \int_{\mathbb{Z}_{p}} B_{k, n}(x) B_{k, m}(x) B_{k, s}(x) d \mu_{-1}(x) \\
& \quad= \begin{cases}2+E_{n+m+s} & \text { if } k=0, \\
\left(\begin{array}{l}
n \\
k
\end{array}\right)\left(\begin{array}{l}
m \\
k
\end{array}\right)\left(\begin{array}{l}
s \\
k
\end{array}\right) \sum_{j=0}^{3 k}\left(\begin{array}{c}
3 k \\
j
\end{array}\right)(-1)^{3 k-j} E_{n+m+s-j} & \text { if } k>0 .\end{cases}
\end{aligned}
$$

By (2.17) and Theorem 2.8, we obtain the following corollary. 
Corollary 2.9. For $m, n, s, k \in \mathbb{Z}_{+}$with $m+n+s>3 k$, one has

$$
\begin{aligned}
& \sum_{j=0}^{n+m+s-3 k}\left(\begin{array}{c}
n+m+s-3 k \\
j
\end{array}\right)(-1)^{j} E_{j+3 k} \\
& = \begin{cases}2+E_{n+m+s} & \text { if } k=0, \\
\sum_{j=0}^{3 k}\left(\begin{array}{c}
3 k \\
j
\end{array}\right)(-1)^{3 k-j} E_{n+m+s-j} & \text { if } k>0 .\end{cases}
\end{aligned}
$$
theorem.

Using the above theorems and mathematical induction, we obtain the following

Theorem 2.10. Let $s \in \mathbb{N}$. For $n_{1}, n_{2}, \ldots, n_{s}, k \in \mathbb{Z}_{+}$with $n_{1}+n_{2}+\cdots+n_{s}>s k$, the multiplication of the sequence of Bernstein polynomials $B_{k, n_{1}}(x), \ldots, B_{k, n_{s}}(x)$ with different degrees under fermionic $p$-adic invariant integral on $\mathbb{Z}_{p}$ can be given as

$$
\int_{\mathbb{Z}_{p}}\left(\prod_{i=1}^{s} B_{k, n_{i}}(x)\right) d \mu_{-1}(x)= \begin{cases}2+E_{n_{1}+n_{2}+\cdots+n_{s}} & \text { if } k=0, \\
\left(\prod_{i=1}^{s}\left(\begin{array}{c}
n_{i} \\
k
\end{array}\right)\right) \sum_{j=0}^{s k}\left(\begin{array}{c}
s k \\
j
\end{array}\right)(-1)^{s k-j} E_{n_{1}+n_{2}+\cdots+n_{s}-j} & \text { if } k>0 .\end{cases}
$$

We also easily see that

$$
\int_{\mathbb{Z}_{p}}\left(\prod_{i=1}^{s} B_{k, n_{i}}(x)\right) d \mu_{-1}(x)=\left(\prod_{i=1}^{s}\left(\begin{array}{l}
n_{i} \\
k
\end{array}\right)\right) \sum_{j=0}^{n_{1}+\cdots+n_{s}-s k}\left(\begin{array}{c}
n_{1}+\cdots+n_{s}-s k \\
j
\end{array}\right)(-1)^{j} E_{j+s k} .
$$

By Theorem 2.10 and (2.22), we obtain the following corollary.

Corollary 2.11. Let $s \in \mathbb{N}$. For $n_{1}, n_{2}, \ldots, n_{s}, k \in \mathbb{Z}_{+}$with $n_{1}+n_{2}+\cdots+n_{s}>s k$, one has

$$
\sum_{j=0}^{n_{1}+\cdots+n_{s}-s k}\left(\begin{array}{c}
n_{1}+\cdots+n_{s}-s k \\
j
\end{array}\right)(-1)^{j} E_{j+s k}= \begin{cases}2+E_{n_{1}+n_{2}+\cdots+n_{s}} & \text { if } k=0 \\
\sum_{j=0}^{s k}\left(\begin{array}{c}
s k \\
j
\end{array}\right)(-1)^{s k-j} E_{n_{1}+n_{2}+\cdots+n_{s}-j} & \text { if } k>0 .\end{cases}
$$


Journal of Inequalities and Applications

Let $m_{1}, \ldots, m_{s}, n_{1}, \ldots, n_{s}, k \in \mathbb{Z}_{+}$with $m_{1} n_{1}+\cdots+m_{s} n_{s}>\left(m_{1}+\cdots+m_{s}\right) k$. By the definition of $B_{k, n_{s}}^{m_{s}}(x)$, we easily get

$$
\begin{aligned}
& \int_{\mathbb{Z}_{p}}\left(\prod_{i=1}^{s} B_{k, n_{i}}^{m_{i}}(x)\right) d \mu_{-1}(x) \\
& =\left(\prod_{i=1}^{s}\left(\begin{array}{c}
n_{i} \\
k
\end{array}\right)^{m_{i}}\right)^{\mathrm{k} \sum_{j=0}^{s} m_{i}}(-1)^{k \sum_{i=1}^{s} m_{i}-j} \int_{\mathbb{Z}_{p}}(1-x)^{\sum_{i=1}^{s} n_{i} m_{i}-j} d \mu_{-1}(x) \\
& =\left(\prod_{i=1}^{s}\left(\begin{array}{c}
n_{i} \\
k
\end{array}\right)^{m_{i}}\right) k \sum_{j=0}^{k \sum_{i=1}^{s} m_{i}}\left(\begin{array}{c}
k \sum_{i=1}^{s} m_{i} \\
j
\end{array}\right)(-1)^{k \sum_{i=1}^{s} m_{i}-j}\left(2+E_{\sum_{i=1}^{s} n_{i} m_{i}-j}\right) \\
& = \begin{cases}2+E_{m_{1} n_{1}+\cdots+m_{s} n_{s}} & \text { if } k=0, \\
\left(\prod_{i=1}^{s}\left(\begin{array}{c}
n_{i} \\
k
\end{array}\right)^{m_{i}}\right) \sum_{j=0}^{k \sum_{i=1}^{s} m_{i}}\left(\begin{array}{c}
k \sum_{i=1}^{s} m_{i} \\
j
\end{array}\right)(-1)^{k \sum_{i=1}^{s} m_{i}-j} E_{\sum_{i=1}^{s} n_{i} m_{i}-j} & \text { if } k>0 .\end{cases}
\end{aligned}
$$

Therefore, we obtain the following theorem.

Theorem 2.12. Let $s \in \mathbb{N}$. For $m_{1}, \ldots, m_{s}, n_{1}, \ldots, n_{s}, k \in \mathbb{Z}_{+}$with $m_{1} n_{1}+\cdots+m_{s} n_{s}>\left(m_{1}+\cdots+\right.$ $\left.m_{s}\right) k$, one has

$$
\begin{aligned}
& \int_{\mathbb{Z}_{p}}\left(\prod_{i=1}^{s} B_{k, n_{i}}^{m_{i}}(x)\right) d \mu_{-1}(x) \text { if } k=0, \\
&= \begin{cases}2+E_{m_{1} n_{1}+\cdots+m_{s} n_{s}} & \\
\left(\prod_{i=1}^{s}\left(\begin{array}{c}
n_{i} \\
k
\end{array}\right)\right)^{m_{i}} \sum_{j=0}^{k \sum_{i=1}^{s} m_{i}}\left(\begin{array}{c}
k \sum_{i=1}^{s} m_{i} \\
j
\end{array}\right)(-1)^{k \sum_{i=1}^{s} m_{i}-j} E_{\sum_{i=1}^{s} n_{i} m_{i}-j} & \text { if } k>0 .\end{cases}
\end{aligned}
$$

By simple calculation, we easily get

$$
\begin{aligned}
& \int_{\mathbb{Z}_{p}}\left(\prod_{i=1}^{s} B_{\mathrm{k}, n_{i}}^{m_{i}}(x)\right) d \mu_{-1}(x) \\
& \quad=\left(\prod_{i=1}^{s}\left(\begin{array}{l}
n_{i} \\
k
\end{array}\right)^{m_{i}}\right)^{\sum_{i=1}^{s} n_{i} m_{i}-k \sum_{j=0}^{s} m_{i}}\left(\sum_{i=1}^{s} n_{i} m_{i}-k \sum_{i=1}^{s} m_{i}\right. \\
& j
\end{aligned}
$$

where $m_{1}, \ldots, m_{s}, n_{1}, \ldots, n_{s}, k \in \mathbb{Z}_{+}$for $s \in \mathbb{N}$. By Theorem 2.12 and (2.26), we obtain the following corollary. 
Corollary 2.13. Let $s \in \mathbb{N}$. For $m_{1}, \ldots, m_{s}, n_{1}, \ldots, n_{s}, k \in \mathbb{Z}_{+}$with $m_{1} n_{1}+\cdots+m_{s} n_{s}>\left(m_{1}+\cdots+\right.$ $\left.m_{s}\right) k$, one has

$$
\begin{aligned}
& \sum_{j=0}^{\sum_{i=1}^{S} n_{i} m_{i}-k} \sum_{i=1}^{S} m_{i}\left(\sum_{i=1}^{S} n_{i} m_{i}-k \sum_{i=1}^{S} m_{i}\right)(-1)^{j} E_{k} \sum_{i=1}^{s} m_{i}-j \\
& = \begin{cases}2+E_{m_{1} n_{1}+\cdots+m_{s} n_{s}} & \text { if } k=0, \\
\sum_{j=0}^{s} \sum_{i=1}^{s} m_{i}\left(\begin{array}{c}
k \sum_{i=1}^{s} m_{i} \\
j
\end{array}\right)(-1)^{k \sum_{i=1}^{s} m_{i}-j} E_{\sum_{i=1}^{s} n_{i} m_{i}-j} & \text { if } k>0 .\end{cases}
\end{aligned}
$$

The fermionic $p$-adic invariant integral of multiplication of $(n+1)$ Bernstein polynomials, the $n$th degree Bernstein polynomials $B_{i, n}(x)$ with $i=0,1, \ldots, n$ and with multiplicity $m_{0}, m_{1}, \ldots, m_{n}$ on $\mathbb{Z}_{p}$, respectively, can be given by

$$
\begin{aligned}
\int_{\mathbb{Z}_{p}}\left(\prod_{i=0}^{n} B_{i, n}^{m_{i}}(x)\right) d \mu_{-1}(x) & =\left(\prod_{i=0}^{n}\left(\begin{array}{c}
n \\
i
\end{array}\right)^{m_{i}}\right) \int_{\mathbb{Z}_{p}} x^{\sum_{i=1}^{n} i m_{i}}(1-x)^{n \sum_{i=0}^{n} m_{i}-\sum_{i=1}^{n} i m_{i}} d \mu_{-1}(x) \\
& =\frac{\left(\prod_{i=1}^{n}\left(\begin{array}{c}
n \\
i
\end{array}\right)^{m_{i}}\right)}{\left(\begin{array}{c}
n \sum_{i=0}^{n} m_{i} \\
\sum_{i=1}^{n} i m_{i}
\end{array}\right)} \int_{\mathbb{Z}_{p}} B_{\sum_{i=1}^{n} i m_{i}, n \sum_{i=0}^{n} m_{i}(x) d \mu_{-1}(x),}
\end{aligned}
$$

where $m_{0}, m_{1}, \ldots, m_{n} \in \mathbb{Z}_{+}$with $n \in \mathbb{Z}_{+}$.

Assume that $n m_{0}+n m_{1}+\cdots+n m_{n}>m_{1}+2 m_{2}+\cdots+n m_{n}$. Then one has

$$
\begin{aligned}
& \int_{\mathbb{Z}_{p}}\left(\prod_{i=0}^{n} B_{i, n}^{m_{i}}(x)\right) d \mu_{-1}(x) \\
& \quad= \begin{cases}2+E_{n m_{0}+n m_{1}+\cdots+n m_{n}} & \text { if } \sum_{i=1}^{n} i m_{i}=0, \\
\left(\prod_{i=0}^{n}\left(\begin{array}{c}
n \\
i
\end{array}\right)^{m_{i}}\right) \sum_{j=0}^{\sum_{i=1}^{n} m_{i}}\left(\begin{array}{c}
n \\
\sum_{i=1}^{n} i m_{i} \\
j
\end{array}\right)(-1)^{\sum_{i=1}^{n} i m_{i}-j} E_{n} \sum_{i=0}^{n} m_{\mathrm{i}}-\sum_{i=1}^{n} i m_{i} & \text { if } \sum_{i=1}^{n} i m_{i}>0 .\end{cases}
\end{aligned}
$$

Therefore, we obtain the following theorem. 
Theorem 2.14. Let $n \in \mathbb{Z}_{+}$.

(i) For $m_{0}, m_{1}, \ldots, m_{n} \in \mathbb{Z}_{+}$with $n \sum_{i=0}^{n} m_{i}>\sum_{i=1}^{n} i m_{i}$, one has

$$
\begin{aligned}
& \int_{\mathbb{Z}_{p}}\left(\prod_{i=0}^{n} B_{i, n}^{m_{i}}(x)\right) d \mu_{-1}(x) \\
& \quad= \begin{cases}2+E_{n m_{0}+n m_{1}+\cdots+n m_{n}} & \text { if } \sum_{i=1}^{n} i m_{i}=0, \\
\left(\prod_{i=0}^{n}\left(\begin{array}{l}
n \\
i
\end{array}\right)\right) \sum_{j=0}^{m_{i}}\left(\begin{array}{c}
\sum_{i=1}^{n} m_{i} \\
j
\end{array}\right)(-1)^{\sum_{i=1}^{n} i m_{i}-j} E_{n} \sum_{i=0}^{n} m_{i}-\sum_{i=1}^{n} i m_{i} & \text { if } \sum_{i=1}^{n} i m_{i}>0 .\end{cases}
\end{aligned}
$$

(ii) For $m_{0}, m_{1}, \ldots, m_{n} \in \mathbb{Z}_{+}$, one has

$$
\left.\int_{\mathbb{Z}_{p}}\left(\prod_{i=0}^{n} B_{i, n}^{m_{i}}(x)\right) d \mu_{-1}(x)=\left(\prod_{i=0}^{n}\left(\begin{array}{l}
n \\
i
\end{array}\right)^{m_{i}}\right)^{n \sum_{i=0}^{n} \sum_{j=0}^{m_{i}-\sum_{i=1}^{n} i m_{i}}\left(n \sum_{i=0}^{n} m_{i}-\sum_{i=1}^{n} i m_{i}\right.}\right)(-1)^{j} E_{\sum_{i=1}^{n} i m_{i}+j} .
$$

By Theorem 2.14, we obtain the following corollary.

Corollary 2.15. For $n, m_{0}, m_{1}, \ldots, m_{n} \in \mathbb{Z}_{+}$with $n \sum_{i=0}^{n} m_{i}>\sum_{i=1}^{n}$ im $m_{i}$, one has

$$
\begin{aligned}
& \sum_{j=0}^{n \sum_{i=0}^{n} m_{i}-\sum_{i=1}^{n} i m_{i}}\left(n \sum_{i=0}^{n} m_{i}-\sum_{i=1}^{n} i m_{i}\right)(-1)^{j} E_{\sum_{i=1}^{n} i m_{i}+j} \\
& = \begin{cases}2+E_{n m_{0}+n m_{1}+\cdots+n m_{n}} & \text { if } \sum_{i=1}^{n} i m_{i}=0, \\
\sum_{j=0}^{n} m_{i}\left(\begin{array}{c}
\sum_{i=1}^{n} i m_{i} \\
j
\end{array}\right)(-1)^{\sum_{i=1}^{n} i m_{i}-j} E_{n \sum_{i=0}^{n} m_{i}-\sum_{i=1}^{n} i m_{i}} & \text { if } \sum_{i=1}^{n} i m_{i}>0 .\end{cases}
\end{aligned}
$$

\section{References}

[1] T. Kim, " $q$-Volkenborn integration," Russian Journal of Mathematical Physics, vol. 9, no. 3, pp. 288-299, 2002.

[2] T. Kim, "Barnes-type multiple $q$-zeta functions and $q$-Euler polynomials," Journal of Physics A, vol. 43, no. 25, Article ID 255201, 11 pages, 2010.

[3] M. Acikgoz and S. Araci, "A study on the integral of the product of several type Bernstein polynomials," IST Transaction of Applied Mathematics-Modelling and Simulation. In press.

[4] S. Bernstein, "Démonstration du théorème de Weierstrass, fondée sur le calcul des probabilities," Communications of the Kharkov Mathematical Society, vol. 13, pp. 1-2, 1912. 
[5] T. Kim, J. Choi, and Y. H. Kim, "On extended carlitz's type q-Euler numbers and polynomials," Advanced Studies in Contemporary Mathematics, vol. 20, no. 4, pp. 499-505, 2010.

[6] N. K. Govil and V. Gupta, "Convergence of $q$-Meyer-König-Zeller-Durrmeyer operators," Advanced Studies in Contemporary Mathematics, vol. 19, no. 1, pp. 97-108, 2009.

[7] V. Gupta, T. Kim, J. Choi, and Y.-H. Kim, “Generating function for $q$-Bernstein, $q$-Meyer-König-Zeller and q-beta basis," Automation Computers Applied Mathematics, vol. 19, pp. 7-11, 2010.

[8] T. Kim, "q-extension of the Euler formula and trigonometric functions," Russian Journal of Mathematical Physics, vol. 14, no. 3, pp. 275-278, 2007.

[9] T. Kim, " $q$-Bernoulli numbers and polynomials associated with Gaussian binomial coefficients," Russian Journal of Mathematical Physics, vol. 15, no. 1, pp. 51-57, 2008.

[10] T. Kim, J. Choi, and Y.-H. Kim, "Some identities on the $q$-Bernstein polynomials, $q$-Stirling numbers and q-Bernoulli numbers," Advanced Studies in Contemporary Mathematics, vol. 20, no. 3, pp. 335-341, 2010.

[11] T. Kim, L.-C. Jang, and H. Yi, “A note on the modified q-bernstein polynomials," Discrete Dynamics in Nature and Society, vol. 2010, Article ID 706483, 12 pages, 2010.

[12] T. Kim, "Note on the Euler q-zeta functions," Journal of Number Theory, vol. 129, no. 7, pp. 1798-1804, 2009.

[13] V. Kurt, "A further symmetric relation on the analogue of the Apostol-Bernoulli and the analogue of the Apostol-Genocchi polynomials," Applied Mathematical Sciences, vol. 3, no. 53-56, pp. 2757-2764, 2009.

[14] I. N. Cangul, V. Kurt, H. Ozden, and Y. Simsek, "On the higher-order $w$ - $q$-Genocchi numbers," Advanced Studies in Contemporary Mathematics, vol. 19, no. 1, pp. 39-57, 2009.

[15] L.-C. Jang, W.-J. Kim, and Y. Simsek, "A study on the $p$-adic integral representation on $\mathbb{Z}_{p}$ associated with Bernstein and Bernoulli polynomials," Advances in Difference Equations, vol. 2010, Article ID 163217, 6 pages, 2010. 